Preprint de "On group strategy-proof mechanisms for a many-to-one maching model," de Ruth Martínez, Jordi Massó, Alejandro Neme i Jorge Oviedo. International Journal of Game Theory 33, 115-128 (2004). Lliurat a Springer l'agost de 2004. 


\title{
On Group Strategy-proof Mechanisms for a Many-to-one Matching Model*
}

\author{
by \\ Ruth Martínez ${ }^{\dagger}$, Jordi Massó $0^{\ddagger}$, Alejandro Neme ${ }^{\dagger}$, and Jorge Oviedo ${ }^{\dagger}$
}

August 2004

\begin{abstract}
For the many-to-one matching model in which firms have substitutable and quota $q$-separable preferences over subsets of workers we show that the workers-optimal stable mechanism is group strategy-proof for the workers. Therefore, in centralized markets like entry-level professional labor markets if the proposed matching is the workers-optimal stable matching then, no group of workers can never benefit by reporting untruthfully their preference relations. We exhibit an example showing that this property fails if the preferences of firms are substitutable but not quota $q$-separable.
\end{abstract}

Journal of Economic Literature Classification Numbers: C78, D71, D78.

Keywords: Matching, Stability, Strategy-proofness.

\footnotetext{
${ }^{*}$ We are grateful to Flip Klijn, Howard Petith, William Thomson, a referee and an associate editor of this journal for helpful comments. The work of R. Martínez, A. Neme, and J. Oviedo is partially supported by the Universidad Nacional de San Luis through Grant 319502, by the Consejo Nacional de Investigaciones Científicas y Técnicas CONICET, through Grant PICT-02114, and by the Agencia Nacional de Promoción Científica y Técnica, through Grant 03-10814. The work of J. Massó is partially supported by the Spanish Ministry of Science and Technology, through Grant BEC2002-2130, and by the Generalitat de Catalunya, through Grant 2001SGR-00162 and the Barcelona Economics Program (CREA). All authors acknowledge financial support from the Grant PCI España-Iberoamérica 2003 (Programa de Cooperación Interuniversitaria de la Agencia Española de Cooperación Internacional-AECI).

${ }^{\dagger}$ Instituto de Matemática Aplicada de San Luis. Universidad Nacional de San Luis and CONICET. Ejército de los Andes 950. 5700, San Luis, Argentina. E-mails: martinez@unsl.edu.ar, aneme@unsl.edu.ar, and joviedo@unsl.edu.ar

†Departament d’Economia i d'Història Econòmica and CODE. Universitat Autònoma de Barcelona. 08193, Bellaterra (Barcelona), Spain. E-mail: jordi.masso@uab.es
} 


\section{Introduction}

Two-sided, many-to-one matching models have been used to study assignment problems where agents can be divided, from the very beginning, into two disjoint subsets: the set of institutions and the set of individuals. The fundamental question of these assignment problems consists of matching each firm, on one side, with a group of workers, on the other side (we follow the convention of generically referring to institutions as firms and to individuals as workers). The problem arises because each firm has preferences over acceptable subsets of workers and each worker has preferences over acceptable firms. Many economic problems with indivisibilities conform to this description. Entry-level professional labor markets and the admission of students to colleges are well-known examples. ${ }^{1} \mathrm{~A}$ matching is called stable if all agents have acceptable partners and there is no unmatched worker-firm pair who both would prefer to be matched to each other rather than staying with their current partners.

The "college admissions model with substitutable preferences" is the name given by Roth and Sotomayor (1990) to the most general many-to-one model with ordinal preferences in which stable matchings exist. Firms are restricted to have substitutable preferences over subsets of workers; namely, all firms want to employ a worker even if other workers become unavailable (Kelso and Crawford (1982) were the first to use this property in a more general model with money). Under this hypothesis the deferred-acceptance algorithms produce either the firms-optimal stable matching or the workers-optimal stable matching, depending on whether the firms or the workers make the offers. The deferred-acceptance algorithm was first proposed by Gale and Shapley (1962) for the marriage model (the one-to-one matching model). It can also be straightforwardly applied to the college admissions problem by reducing it to the marriage model. ${ }^{2}$ The firms (workers)-optimal stable matching is unanimously considered by all firms (respectively, workers) to be the best among all stable matchings.

A more specific many-to-one model, called the "college admissions problem" by Gale and Shapley (1962), supposes that firms have a maximum number of positions to be filled (their quota), and that each firm, given its ranking of individual workers, orders subsets

\footnotetext{
${ }^{1}$ See Roth and Sotomayor (1990) for a detailed description of these two problems as well as for a masterful presentation and analysis of two-sided matching models.

${ }^{2}$ Roth and Sotomayor (Section 6.1, 1990) adapted the algorithm to the more general many-to-one model with substitutable preferences; this adapted version is the one that we will present in Section 2 and we will use in the proof of the Proposition.
} 
of workers in a "responsive" manner; namely, for any two subsets that differ in only one student, a college prefers the subset containing the most-preferred student. In this model the set of stable matchings satisfies many desirable properties (observe that the marriage model is a particular instance of the "college admissions problem" when all firms have quota one). The first type of properties are more theoretical in nature and are related with its lattice structure. The second type of properties have more practical implications and they are related with the strategic incentives of agents participating in centralized markets. In these markets, a mechanism requires each agent to report a preference and associates a matching with the reported preference profile (a mechanism is stable if it selects, for each preference profile, a stable matching). Roth (1984, 1986, 1990, and 1991), Mongell and Roth (1991), Roth and Xing (1994), and Romero-Medina (1998) are examples of papers studying these incentives in particular matching problems like entry-level professional labor markets, student admissions at colleges, and American sororities. However, whether or not a matching is stable depends on the preferences of agents and, since they are private information, agents have to be asked for them; hence, untruthful reports might arise. This is the reason why the matching literature has intensively studied the strategic properties of stable mechanisms. In particular, Dubins and Freedman (1981) shows that in the "college admissions problem" the deferred-acceptance algorithm in which workers make offers is group strategy-proof for the workers. ${ }^{3}$ This means that for the mechanism that selects for each preference profile its corresponding workers-optimal stable matching, no group of workers can never benefit by reporting untruthfully their preference relations. This is an important property and it becomes critical if the market has to be redesigned, in which case the declared preference profile conveys very valuable information.

It is known that this group strategy-proofness property is not necessarily true when the preferences of firms are substitutable. The purpose of this paper is to consider a weaker condition than responsiveness, called quota $q$-separability, that together with substitutability implies that the property that the workers-optimal stable mechanism is group strategyproof for the workers holds for this more general many-to-one matching model. We have

\footnotetext{
${ }^{3}$ To be precise, they show it for the marriage model, but their result can be extended to the college admissions problem. Some results concerning stability in the college admissions problem are immediate consequences of the fact that they hold for the marriage model. Each college is split into as many pieces as positions it has, so transforming the original many-to-one model into a one-to-one model. Responsiveness allows then the translation of stability from one model to another. See Roth and Sotomayor (1990) for a complete description of this procedure as well as for its applications. Observe that this reduction is possible only if preferences are responsive.
} 
already showed that if firms have substitutable and quota $q$-separable preferences then, (a) the set of unmatched agents is the same in all stable matchings (Martínez, Massó, Neme, and Oviedo, 2000) and (b) the set of stable matchings has a lattice structure with respect to two natural binary operations (Martínez, Massó, Neme, and Oviedo, 2001). A firm is said to have "separable" preferences over subsets of workers if its partition between acceptable and unacceptable workers has the property that only adding acceptable workers makes any given subset of workers a better one. However, in many applications such as the entry-level professional labor markets, separability does not seem very reasonable because firms usually have fewer openings (their quota) than the number of "good" workers looking for a job. In these cases it seems reasonable to restrict the preferences of firms in such a way that the separability condition operates only up to their quota, considering unacceptable all subsets with higher cardinality. Moreover, while responsiveness seems to be the relevant property for extending an ordered list of individual students to preferences over all subsets of students, it is too restrictive to capture some degree of complementarity among workers, which can be very natural in other settings. The quota $q$-separability condition permits greater flexibility in going from orders on individuals to orders on subsets, making the model more applicable. For instance, candidates for a job can be grouped together by areas of specialization. A firm with quota two may consider as the best subset of workers not the set consisting of the first two candidates according to its ranking of individuals (they may have both the same specialization) but rather the subset composed of the first and fourth candidates in the individual ranking (i.e.; the first in each area of specialization).

As is the case for the college admissions problem, the proof of the property that (in this more general many-to-one matching model) the workers-optimal stable mechanism is group strategy-proof for the workers uses the following result (known in the literature as the Blocking Lemma): Suppose that the set of workers who strictly prefer an individually rational matching to the workers-optimal stable matching is nonempty. Then, we can always find a firm and a worker (a blocking pair of the individually rational matching) with the following properties: (a) the firm was hiring another worker who strictly prefers the individually rational matching to the workers-optimal stable matching and (b) the worker (member of the blocking pair) considers the workers-optimal stable matching to be at least as good as the individually rational matching. Furthermore, and in order to prove the Blocking Lemma, we also show that the workers-optimal stable matching is weakly Pareto optimal for the workers; namely, there is no individually rational matching that all workers strictly prefer to the workers-optimal stable matching. 
Since our many-to-one matching model includes (as a particular subclass) the college admissions problem, all negative results concerning strategic incentives of agents of the latter model carry over to the former one. In particular, the workers-optimal stable mechanism is not group strategy-proof for the firms (it is not even strategy-proof for them) and there is no stable and strategy-proof mechanism. In addition, the workers-optimal stable mechanism is, on the domain of substitutable and quota $q$-separable preference profiles, the unique stable and group strategy-proof mechanism for the workers since it is already the unique one in the college admissions problem.

The paper is organized as follows. In Section 2, we present the preliminary notation and definitions. In Section 3, we present our results for the many-to-one matching model in which firms have substitutable and quota $q$-separable preferences. We state that the workers-optimal stable mechanism is group strategy-proof for the workers (Theorem) and that the workers-optimal stable matching is weakly Pareto optimal for the workers (Proposition). Furthermore, we state (without proof) that the Blocking Lemma holds as well. In Section 4, we exhibit an example showing that none of these three results hold if the preferences of firms are substitutable but not quota $q$-separable. Finally, the Appendix in Section 5 contains the proof of the Theorem and the Proposition.

\section{Preliminaries}

There are two disjoint sets of agents, a set of $n$ firms $F=\left\{f_{1}, \ldots, f_{n}\right\}$ and a set of $m$ workers $W=\left\{w_{1}, \ldots, w_{m}\right\}$. Generic elements of both sets are denoted, respectively, by $f, \bar{f}$, and $\tilde{f}$, and by $w, \bar{w}$, and $\widetilde{w}$. Each worker $w \in W$ has a strict, transitive, and complete preference relation $P(w)$ over $F \cup\{\emptyset\}$, and each firm $f \in F$ has a strict, transitive, and complete preference relation $P(f)$ over $2^{W}$. Preference profiles are $(n+m)$-tuples of preference relations and they are represented by $P=\left(P\left(f_{1}\right), \ldots, P\left(f_{n}\right) ; P\left(w_{1}\right), \ldots, P\left(w_{m}\right)\right)$. Given a preference relation of a firm $P(f)$, the subsets of workers preferred to the empty set by $f$ are called acceptable. Similarly, given a preference relation of a worker $P(w)$, the firms preferred by $w$ to the empty set are called acceptable. Therefore, we allow for the possibility that a firm may prefer not to hire any worker rather than to hire unacceptable subsets of workers and that a worker may prefer to remain unemployed rather than to work for an unacceptable firm. To describe preference relations in a concise manner, and since only acceptable partners matter, we write acceptable partners in the order of decreasing 
preference. For instance,

$$
\begin{aligned}
& P\left(f_{i}\right) \quad: \quad w_{1} w_{3}, w_{2}, w_{1} \\
& P\left(w_{j}\right): f_{1}, f_{3}
\end{aligned}
$$

means that $\left\{w_{1}, w_{3}\right\} P\left(f_{i}\right)\left\{w_{2}\right\} P\left(f_{i}\right)\left\{w_{1}\right\} P\left(f_{i}\right) \emptyset$ and $f_{1} P\left(w_{j}\right) f_{3} P\left(w_{j}\right) \emptyset$.

A market is a triple $(F, W, P)$, where $F$ is a set of firms, $W$ is a set of workers, and $P$ is a preference profile. Given a market $(F, W, P)$ the assignment problem consists of matching workers with firms, keeping the bilateral nature of their relationship and allowing for the possibility that both firms and workers may remain unmatched. Formally,

Definition 1 A matching $\mu$ is a mapping from the set $F \cup W$ into the set of all subsets of $F \cup W$ such that for all $w \in W$ and all $f \in F$ :

1. Either $|\mu(w)|=1$ and $\mu(w) \subseteq F$ or else $\mu(w)=\emptyset$.

2. $\mu(f) \in 2^{W}$.

3. $\mu(w)=\{f\}$ if and only if $w \in \mu(f)$.

Condition 1 says that a worker is either matched to at most one firm, or remains unmatched. Condition 2 says that a firm either hires a subset of workers, or is unmatched. Finally, condition 3 states the bilateral nature of a matching: firm $f$ hires worker $w$ if and only if worker $w$ works for firm $f$. We say that $f$ is unmatched in a matching $\mu$ if $\mu(f)=\emptyset$ and that $w$ is unmatched in a matching $\mu$ if $\mu(w)=\emptyset$. Otherwise, they are matched. A matching $\mu$ is said to be one-to-one if each firm hires at most one worker; namely, Condition 2 is replaced by: Either $|\mu(f)|=1$ and $\mu(f) \subseteq W$ or else $\mu(f)=\emptyset$. The model in which all matchings are one-to-one is also known as the marriage model. The model in which all matchings are many-to-one (i.e., satisfy Definition 1), and firms have responsive preferences, ${ }^{4}$ is known as the college admissions problem (Gale and Shapley, 1962). To represent matchings concisely we follow the widespread notation where, for instance, given $F=\left\{f_{1}, f_{2}, f_{3}\right\}$ and $W=\left\{w_{1}, w_{2}, w_{3}, w_{4}\right\}$,

$\begin{array}{ccccc} & f_{1} & f_{2} & f_{3} & \emptyset \\ \mu & w_{3} w_{4} & w_{1} & \emptyset & w_{2}\end{array}$

\footnotetext{
${ }^{4}$ Roughly, for any two subsets of workers that differ in only one worker a firm prefers the subset containing the most-preferred worker. See Roth and Sotomayor (1990) for a precise and formal definition of responsive preferences.
} 
means that firm $f_{1}$ is matched to workers $w_{3}$ and $w_{4}$, firm $f_{2}$ is matched to worker $w_{1}$, and firm $f_{3}$ and worker $w_{2}$ are unmatched. Given a matching $\mu$ and two subsets $F^{\prime} \subseteq F$ and $W^{\prime} \subseteq W$ we denote by $\mu\left(F^{\prime}\right)$ and $\mu\left(W^{\prime}\right)$ the sets $\left\{w \in W \mid \mu(w) \in F^{\prime}\right\}$ and $\left\{f \in F \mid \exists w \in W^{\prime}\right.$ such that $\left.w \in \mu(f)\right\}$, respectively.

Let $P$ be a preference profile. Given a set of workers $S \subseteq W$, let $C h(S, P(f))$ denote firm f's most-preferred subset of $S$ according to its preference ordering $P(f)$. Generically we refer to this set as the choice set.

A matching $\mu$ is blocked by worker $w$ if $\emptyset P(w) \mu(w)$. A matching $\mu$ is blocked by firm $f$ if $\mu(f) \neq C h(\mu(f), P(f))$. A matching is individually rational if it is not blocked by any individual agent. We denote by $\operatorname{IR}(P)$ the set of individually rational matchings. A matching $\mu$ is blocked by a firm-worker pair $(f, w)$ if $w \notin \mu(f), w \in C h(\mu(f) \cup\{w\}, P(f))$, and $f P(w) \mu(w)$.

Definition 2 A matching $\mu$ is stable if it is not blocked by any individual agent or any firm-worker pair.

Given a preference profile $P$, we denote the set of stable matchings by $S(P)$. It is easy to construct examples of preference profiles with the property that the set of stable matchings is empty. These examples share the feature that at least one firm regards a subset of workers as complements. This is the reason why the literature has focused on the restriction where workers are regarded as substitutes.

Definition 3 A firm $f$ 's preference relation $P(f)$ satisfies substitutability if for any set $S$ containing workers $w$ and $w^{\prime}\left(w \neq w^{\prime}\right)$, if $w \in C h(S, P(f))$ then $w \in C h\left(S \backslash\left\{w^{\prime}\right\}, P(f)\right)$.

A preference profile $P$ is substitutable if for each firm $f$, the preference relation $P(f)$ satisfies substitutability. Kelso and Crawford (1982) shows that (in a more general model with money) if all firms have substitutable preferences then: (1) the set of stable matchings is non-empty, and (2) firms unanimously agree that a stable matching $\mu_{F}$ is the best stable matching. Roth (1984) extends these results and shows that if all firms have substitutable preferences then: (3) workers unanimously agree that a stable matching $\mu_{W}$ is the best stable matching, ${ }^{5}$ and (4) the optimal stable matching for one side is the worst stable

\footnotetext{
${ }^{5}$ The matchings $\mu_{F}$ and $\mu_{W}$ are called, respectively, the firms-optimal stable matching and the workersoptimal stable matching. We are following the convention of extending preferences from the original sets $\left(2^{W}\right.$ and $\left.F \cup\{\emptyset\}\right)$ to the set of matchings. However, we now have to consider weak preference relations
} 
matching for the other side. That is, $S(P) \neq \emptyset$ and for all $\mu \in S(P)$ we have that $\mu_{F} R(f) \mu R(f) \mu_{W}$ for all $f \in F$ and $\mu_{W} R(w) \mu R(w) \mu_{F}$ for all $w \in W$.

The deferred-acceptance algorithm, originally defined by Gale and Shapley (1962) for the marriage model, produces either $\mu_{F}$ or $\mu_{W}$ depending on who makes the offers. At any step of the algorithm in which firms make offers, a firm proposes itself to the choice set of the set of workers who have not already rejected it during the previous steps, while a worker accepts the offer of the best firm among the set of current offers plus the one made by the firm provisionally matched in the previous step (if any). The algorithm stops at the step at which all offers are accepted; the (provisional) matching then becomes definite and it is the firms-optimal stable matching $\mu_{F}$. Symmetrically, at any step of the algorithm in which workers make offers, a worker proposes himself to the best firm among the set of firms that have not already rejected him during the previous steps, while a firm accepts the choice set of the set of current offers plus that of the workers provisionally matched in the previous step (if any). The algorithm stops at the step at which all offers are accepted; the (provisional) matching then becomes definite and it is the workers-optimal stable matching $\mu_{W}$.

A firm $f$ has separable preferences if the division between good workers $(\{w\} P(f) \emptyset)$ and bad workers $(\emptyset P(f)\{w\})$ guides the ordering of subsets in the sense that adding a good worker leads to a better set, while adding a bad worker leads to a worse set. ${ }^{6}$ Formally,

Definition 4 A firm $f$ 's preference relation $P(f)$ satisfies separability if for all $S \subseteq W$ and $w \notin S$ we have that $(S \cup\{w\}) P(f) S$ if and only if $\{w\} P(f) \emptyset$.

A preference profile $P$ is separable if for each firm $f$, the preference relation $P(f)$ satisfies separability.

Remark 1 All separable preference relations are substitutable. To see this, just note that if $P(f)$ is separable then, for every $S \subseteq W, C h(S, P(f))=\{w \in S \mid\{w\} P(f) \emptyset\}$. Moreover,

since the matchings $\mu$ and $\mu^{\prime}$ may associate to an agent the same partner. These preference relations will be denoted by $R(f)$ and $R(w)$. For instance, to say that all firms prefer $\mu_{F}$ to any stable $\mu$ means that for every $f \in F$ we have that $\mu_{F} R(f) \mu$ for all stable $\mu$ (that is, either $\mu_{F}(f)=\mu(f)$ or else $\mu_{F}(f) P(f) \mu(f)$ ).

${ }^{6}$ This condition has been extensively used in social choice; see, for instance, Barberà, Sonnenschein, and Zhou (1991). In the matching literature, Sönmez (1996), Dutta and Massó (1997), Martínez, Massó, Neme, and Oviedo (2000) and (2001) have used it to study, respectively, strategy-proof implementation, the stability of matchings when workers also care about their colleagues, the set of unmatched agents in different stable matchings, and the lattice structure of the set of stable matchings. 
the preference relation

$$
P(f): w_{1}, w_{1} w_{2}, w_{2}
$$

shows that not all substitutable preference relations are separable.

Sönmez (1996) shows that if firms have separable preferences then there exists a unique stable matching. A simple way to construct this unique stable matching $\mu$ is as follows: for each $w \in W$, let $\mu(w)$ be the maximal element, according to $P(w)$, on the set of firms for which $w$ is an acceptable worker, i.e., $\{f \in F \mid\{w\} P(f) \emptyset\}$. The stability of $\mu$ follows directly from separability of firms' preferences.

Here, we assume that each firm $f$ has, in addition to substitutable and separable preferences, a maximum number of positions to be filled: its quota $q_{f}$. This limitation may arise from, for example, technological, legal, or budgetary reasons. Since we are interested in stable matchings, we introduce this restriction by incorporating it into the preference relation of the firm. The college admissions model with responsive preferences (Gale and Shapley, 1962) incorporates the quota restriction of each college by imposing a limit on the number of students that a college may admit . However, from the point of view of stability, this is equivalent to supposing that all sets of students with cardinality larger than the quota are unacceptable for the college. Therefore, even if the number of good workers for firm $f$ is larger than its quota $q_{f}$, all sets of workers with cardinality strictly larger than $q_{f}$ will be unacceptable. Formally,

Definition 5 A firm $f$ 's preference relation $P(f)$ over sets of workers is $q_{f}$-separable if: (a) for all $S \subsetneq W$ such that $|S|<q_{f}$ and $w \notin S$ we have that $(S \cup\{w\}) P(f) S$ if and only if $\{w\} P(f) \emptyset$, and $(b) \emptyset P(f) S$ for all $S$ such that $|S|>q_{f} \cdot{ }^{7}$

We denote by $q=\left(q_{f}\right)_{f \in F}$ the list of quotas and we say that a preference profile $P$ is quota $q$-separable if each $P(f)$ is quota $q_{f}$-separable. In principle firms may have different quotas. The case where all firms have quota 1 -separable preferences is equivalent, from the point of view of the set of stable matchings, to the marriage model. Hence, our set-up includes the marriage model as a particular case.

\footnotetext{
${ }^{7}$ For the purpose of studying the set of stable matchings, condition (b) in this definition could be replaced by the following condition: $|C h(S, P(f))| \leq q_{f}$ for all $S$ such that $|S|>q_{f}$. We choose condition (b) since it is simpler. Sönmez (1996) uses an alternative approach which consists in deleting condition (b) in the definition but then requiring in the definition of a matching that $|\mu(f)| \leq q_{f}$ for all $f \in F$. Notice that then the set of separable preferences is quota $q_{f}$-separable for all $q_{f}$.
} 
The two preference relations over $2^{\left\{w_{1}, w_{2}, w_{3}\right\}}$

$$
\begin{aligned}
& P(f): w_{1} w_{2}, w_{1} w_{3}, w_{2} w_{3}, w_{1}, w_{2}, w_{3} \\
& P(\bar{f}): w_{1} w_{2} w_{3}, w_{1} w_{2}, w_{1} w_{3}, w_{2} w_{3}, w_{1}, w_{2}, w_{3}
\end{aligned}
$$

illustrate the fact that, in general and given a list of quotas $q$, the sets of separable and quota $q$-separable preferences are unrelated. Firm $f$ 's preference relation is 2 -separable but not separable, since $\emptyset P(f)\left\{w_{1}, w_{2}, w_{3}\right\}$ and all workers are good, while firm $\bar{f}$ 's preference relation is separable but not quota 2 -separable.

Moreover, the preference relation over $2^{\left\{w_{1}, w_{2}, w_{3}, w_{4}\right\}}$

$$
P(f): w_{1} w_{2}, w_{3} w_{4}, w_{1} w_{3}, w_{1} w_{4}, w_{2} w_{3}, w_{2} w_{4}, w_{1}, w_{2}, w_{3}, w_{4}
$$

illustrates the fact that quota $q$-separability does not imply substitutability. To see this, notice that the preference relation $P(f)$ is quota 2-separable but it is not substitutable since $w_{1} \in C h\left(\left\{w_{1}, w_{2}, w_{3}, w_{4}\right\}, P(f)\right)=\left\{w_{1}, w_{2}\right\}$, but $w_{1} \notin C h\left(\left\{w_{1}, w_{3}, w_{4}\right\}, P(f)\right)=$ $\left\{w_{3}, w_{4}\right\}$. However, it is easy to see that all quota $(m-1)$-separable preferences are substitutable.

The preference relation $P(f)$ over $2^{\left\{w_{1}, w_{2}, w_{3}\right\}}$

$$
P(f): w_{1} w_{2} w_{3}, w_{1} w_{3}, w_{1} w_{2}, w_{2} w_{3}, w_{1}, w_{2}, w_{3}
$$

illustrates the fact that the set of responsive preferences is a strict subset of the set of quota $q_{f}$-separable and substitutable preferences, since $P(f)$ is quota 3 -separable and substitutable but it is not responsive because $\left\{w_{1}, w_{3}\right\} P(f)\left\{w_{1}, w_{2}\right\}$ but $\left\{w_{2}\right\} P(f)\left\{w_{3}\right\}$.

The following example shows that even if all firms have quota $q$-separable preferences the set of stable matchings may be empty.

Example 1 Let $F=\left\{f_{1}, f_{2}\right\}$ and $W=\left\{w_{1}, w_{2}, w_{3}, w_{4}\right\}$ be the two sets of agents with the preference profile $P$, where

$$
\begin{aligned}
P\left(f_{1}\right) & : w_{3} w_{4}, w_{2} w_{4}, w_{1} w_{2}, w_{1} w_{3}, w_{2} w_{3}, w_{1} w_{4}, w_{1}, w_{2}, w_{3}, w_{4}, \\
P\left(f_{2}\right) & : w_{3}, w_{4} \\
P\left(w_{1}\right) & : f_{1} \\
P\left(w_{2}\right) & : f_{1} \\
P\left(w_{3}\right) & : f_{1}, f_{2} \\
P\left(w_{4}\right) & : f_{2}, f_{1} .
\end{aligned}
$$


Notice that $P$ is quota $(2,1)$-separable. However, $P\left(f_{1}\right)$ is not substitutable since $w_{3} \in$ $C h\left(W, P\left(f_{1}\right)\right)$ but $w_{3} \notin C h\left(W \backslash\left\{w_{4}\right\}, P\left(f_{1}\right)\right)$. It can be verified that $S(P)=\emptyset$.

The Venn diagram of Figure 1 summarizes these relations.

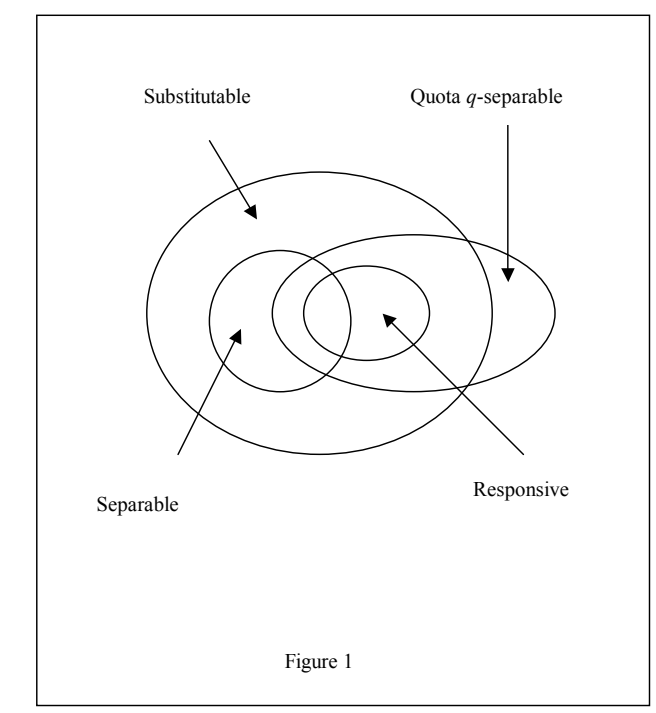

\section{Results}

Some markets are decentralized: workers and firms are themselves responsible for looking for acceptable partners. However, in many assignment problems, like entry-level professional labor markets and students admissions at colleges, matches are proposed by centralized stable procedures (in some markets they are suggested while in others they are imposed). Whether or not a matching is stable depends on the preference profile. But preferences are private information. Therefore, a centralized market consists of a clearing-house that, after asking each agent to report a preference relation, proposes a matching. This defines a mechanism. Formally, let $\widehat{\mathcal{P}}$ be a domain of preference profiles and let $\mathcal{M}$ be the set of all matchings. A mechanism $h: \widehat{\mathcal{P}} \rightarrow \mathcal{M}$ maps each preference profile $P \in \widehat{\mathcal{P}}$ to a matching $h(P) \in \mathcal{M}$. Therefore, $h(P)(f)$ is the set of workers assigned to $f$ and $h(P)(w)$ is the firm assigned to $w$ (if any) at preference profile $P \in \widehat{\mathcal{P}}$ by mechanism $h$. A mechanism $h: \widehat{\mathcal{P}} \rightarrow \mathcal{M}$ is stable if for all $P \in \widehat{\mathcal{P}}, h(P) \in S(P)$.

Let $\mathcal{S}$ be the set of substitutable and quota $q$-separable preference relations of firms over $2^{W}$ and let $\mathcal{T}$ be the set of all preference relations of workers over $F \cup\{\emptyset\}$. The set of all substitutable and quota $q$-separable preference profiles can be written as the 
set $\mathcal{P}=\mathcal{S}^{n} \times \mathcal{T}^{m}$, where $n$ and $m$ are the number of firms and workers, respectively. To emphasize the role of a subset of workers $\widehat{W}$ we write the preference profile $P$ as $\left(P_{\widehat{W}}, P_{-\widehat{W}}\right)$. Therefore, given $\widehat{W} \subseteq W, P \in \mathcal{P}$, and $\widehat{P}_{\widehat{W}} \in \mathcal{T}|\widehat{W}|$ we write $\left(\widehat{P}_{\widehat{W}}, P_{-\widehat{W}}\right)$ to denote the preference profile $P$ where the preference relations $\left.P_{\widehat{W}} \in \mathcal{T}\right|^{\mid \widehat{W}} \mid$ have been replaced by $\widehat{P}_{\widehat{W}} \in$ $\mathcal{T}|\widehat{W}|$. Mechanisms require each agent to report some preference relation. A mechanism is manipulable by a group of workers if its members can obtain better partners by revealing their preference relations untruthfully. A mechanism is group strategy-proof for the workers if it is not manipulable by any group of workers. Formally,

Definition 6 A mechanism $h: \widehat{\mathcal{P}} \rightarrow \mathcal{M}$ is group strategy-proof for the workers if for all preference profiles $P \in \mathcal{P}$, all subsets of workers $\widehat{W} \subseteq W$, all reports $\widehat{P}_{\widehat{W}} \in \mathcal{T}|\widehat{W}|$, and for all $w \in \widehat{W}$,

$$
h(P)(w) R(w) h\left(\widehat{P}_{\widehat{W}}, P_{-\widehat{W}}\right)(w)
$$

Assume that $\widehat{\mathcal{P}}$ is a subset of substitutable preference profiles. We say that $h: \widehat{\mathcal{P}} \rightarrow \mathcal{M}$ is the workers-optimal stable mechanism if it always selects the workers-optimal stable matching; that is, for all $P \in \widehat{\mathcal{P}}, h(P)$ is the workers-optimal stable matching relative to $P$. We are now ready to state the main result of the paper.

Theorem (Group Strategy-proofness) On the domain of substitutable and quota q-separable preference profiles, the workers-optimal stable mechanism $h: \mathcal{P} \rightarrow \mathcal{M}$ is group strategyproof for the workers.

Proof See the Appendix.

The proof of the Theorem is based on an extension of a result (known in the literature as the Blocking Lemma) whose proof uses the fact that the workers-optimal stable matching satisfies a weak Pareto optimality property. We now explain and present these two results.

For the marriage model, Roth (1982) shows that optimal stable matchings have an even stronger optimality property: there is no individually rational matching that all agents of one side of the market strictly prefer to their corresponding optimal stable matching. Roth (1985) partly extends this result to the college admissions problem. He shows (using the result concerning the marriage model) that this weak Pareto optimality property holds for the students (the "one side" of the market); moreover, he also shows that the property in general fails for the colleges (the "many side" of the market). In the next section we exhibit an example (Example 2) where firms have substitutable preferences and the weak Pareto 
optimality property does not even hold for the workers; that is, there is an individually rational matching strictly preferred by all workers (our "one side" of the market) to the workers-optimal stable matching. However, the proposition below states that if firms preferences are also quota $q$-separable, the weak Pareto optimality for the workers is recovered. In our case, though, the proof is genuinely many-to-one since it can not be based on the fact that the result holds for the marriage model.

Proposition (Weak Pareto Optimality) Let $P$ be a substitutable and quota $q$-separable preference profile. Then, there is no individually rational matching $\mu$ such that $\mu(w) P(w) \mu_{W}(w)$ for all $w \in W$.

Proof See the Appendix.

In the proof of the Theorem (as is the case for the marriage model), the Blocking Lemma plays a fundamental role. This lemma states that if the set of workers who strictly prefer an individually rational matching $\mu$ to $\mu_{W}$ is nonempty then, we can always find a blocking pair $(f, w)$ of $\mu$ with the property that $f$ was hiring at $\mu$ a worker strictly preferring $\mu$ to $\mu_{W}$ and $w$ considers $\mu_{W}$ being at least as good as $\mu$. Gale and Sotomayor (1985) proved the Blocking Lemma for the marriage model. Next, we state that a Blocking Lemma also holds for the more general many-to-one model with substitutable and quota $q$-separable preferences. $^{8}$

Lemma (Blocking Lemma) Let $P$ be a substitutable and quota $q$-separable preference profile and let $\mu \in I R(P)$. Denote by $W^{\prime}=\left\{w \in W \mid \mu(w) P(w) \mu_{W}(w)\right\}$ the set of workers who strictly prefer $\mu$ to $\mu_{W}$. If $W^{\prime}$ is nonempty, then there exist $f \in \mu\left(W^{\prime}\right)$ and $w \in W \backslash W^{\prime}$ such that the pair $(f, w)$ blocks $\mu$.

The proof of the Blocking Lemma is very involved and long; therefore, it is omitted (see Martínez, Massó, Neme, and Oviedo (2004) for the complete proof). Again, observe that the Proposition plays a key role in its proof by guaranteeing that $W \backslash W^{\prime} \neq \emptyset$.

\section{Example}

Example 2 below shows that the conclusions of the Theorem, the Proposition and the Lemma are all false without the quota $q$-separability assumption.

\footnotetext{
${ }^{8}$ In the marriage model the Blocking Lemma also plays a fundamental role in the proof of the Strong Stability Theorem of Demange, Gale, and Sotomayor (1987).
} 
Example 2 Let $F=\left\{f_{1}, f_{2}, f_{3}\right\}$ and $W=\left\{w_{1}, w_{2}, w_{3}, w_{4}\right\}$ be the two sets of agents with the substitutable preference profile $P$ such that

$$
\begin{array}{ll}
P\left(f_{1}\right): & w_{1} w_{2}, w_{2}, w_{1}, w_{4}, \\
P\left(f_{2}\right): & w_{3}, w_{2} w_{4}, w_{1} w_{2}, w_{4}, w_{1}, w_{2}, \\
P\left(f_{3}\right): & w_{4}, w_{1}, w_{3}, \\
P\left(w_{1}\right): & f_{2}, f_{3}, f_{1}, \\
P\left(w_{2}\right): & f_{2}, f_{1}, \\
P\left(w_{3}\right): & f_{3}, f_{2}, \\
P\left(w_{4}\right): & f_{2}, f_{1}, f_{3} .
\end{array}
$$

The workers-optimal stable matching is

$$
\begin{array}{cccc} 
& f_{1} & f_{2} & f_{3} \\
\mu_{W} & w_{1} w_{2} & w_{3} & w_{4} .
\end{array}
$$

The individually rational matching

$$
\begin{array}{cccc} 
& f_{1} & f_{2} & f_{3} \\
& w_{4} & w_{1} w_{2} & w_{3}
\end{array}
$$

has the property that $\mu(w) P(w) \mu_{W}(w)$ for all $w \in W$. Hence, the conclusion of the Proposition fails without the quota $q$-separability assumption. The conclusion of the Blocking Lemma also fails because $\mu$ is not stable since the (unique) pair $\left(f_{2}, w_{4}\right)$ blocks $\mu$, but $w_{4} \in W^{\prime}$ because $\mu\left(w_{4}\right)=f_{1} P\left(w_{4}\right) f_{3}=\mu_{W}\left(w_{4}\right)$. Finally, consider the (substitutable) preference relations $\widehat{P}_{W} \in \mathcal{T}^{4}$, where

$$
\begin{array}{lll}
\widehat{P}\left(w_{1}\right): & f_{2}, \\
\widehat{P}\left(w_{2}\right): & f_{2}, \\
\widehat{P}\left(w_{3}\right): & f_{3}, \\
\widehat{P}\left(w_{4}\right): & f_{1} .
\end{array}
$$

Let $h$ be the workers-optimal stable mechanism defined on the domain of substitutable preference profiles. Then, $h\left(\widehat{P}_{W}, P_{-W}\right)=\mu P(w) \mu_{W}=h(P)$ for all $w \in W$, implying that $h$ is not group strategy-proof for the workers on the domain of only substitutable preferences. 


\section{Appendix}

Proof of the Proposition Let $P$ be a substitutable and quota $q$-separable preference profile and assume that $\mu \in I R(P)$ and

$$
\mu(w) P(w) \mu_{W}(w) \text { for all } w \in W
$$

Since $\mu_{W}$ is also individually rational, $\mu(w) \neq \emptyset$ for all $w \in W$. Therefore, $\mu(W)$ is nonempty.

Claim A $\mu(W)=\mu_{W}(W)$.

Proof Let $f \in \mu(W)$. Then $f=\mu(w)$ for some $w$. Observe that $\mu_{W}(f) \in 2^{W} \backslash\{\emptyset\}$. Otherwise $\left(\mu_{W}(f)=\emptyset\right)$, by the individual rationality of $\mu$ and the quota $q_{f}$-separability of $P(f),\{w\} P(f) \emptyset$, implying that $(f, w)$ blocks $\mu_{W}$, contradicting its stability. Thus,

$$
\mu(W) \subseteq \mu_{W}(W)
$$

Since all workers are matched at $\mu$, we already know that $\sum_{f \in \mu(W)}|\mu(f)|=|W|$. To see that the same property holds for $\mu_{W}$, assume $\sum_{f \in \mu(W)}\left|\mu_{W}(f)\right|<|W|$. Thus, there exists $\bar{f} \in F$ such that $\left|\mu_{W}(\bar{f})\right|<|\mu(\bar{f})| \leq q_{\bar{f}}$, implying that we can find $\bar{w} \in \mu(\bar{f}) \backslash \mu_{W}(\bar{f})$. Since $\mu \in I R(P), \bar{w} \in C h(\mu(\bar{f}), P(\bar{f}))$, yielding, by the quota $q_{\bar{f}}$-separability of $P(\bar{f})$, $\{\bar{w}\} P(\bar{f}) \emptyset$. Thus, because $\left|\mu_{W}(\bar{f})\right|<q_{\bar{f}}$,

$$
\bar{w} \in C h\left(\mu_{W}(\bar{f}) \cup\{\bar{w}\}, P(\bar{f})\right) .
$$

The fact that $\bar{f}=\mu(\bar{w}) P(\bar{w}) \mu_{W}(\bar{w})$ and (3) imply that $(\bar{f}, \bar{w})$ blocks $\mu_{W}$, contradicting its stability. Thus,

$$
\sum_{f \in \mu(W)}\left|\mu_{W}(f)\right|=|W|
$$

Hence, $|W| \geq \sum_{f \in F}\left|\mu_{W}(f)\right|=\sum_{f \in \mu(W)}\left|\mu_{W}(f)\right|+\sum_{f \in F \backslash \mu(W)}\left|\mu_{W}(f)\right|$, implying, by (4), that $\sum_{f \in F \backslash \mu(W)}\left|\mu_{W}(f)\right|=0$. Thus, if $f \in F \backslash \mu(W)$ then $\mu_{W}(f)=\emptyset$, which means that $f \notin \mu_{W}(W)$, implying that $F \backslash \mu(W) \subseteq F \backslash \mu_{W}(W)$. Hence, $\mu_{W}(W) \subseteq \mu(W)$ and, by (2),

$$
\mu(W)=\mu_{W}(W)
$$

Claim A is proved.

Claim B $\mu_{W}(\mu(W))=W$. 
Proof Assume otherwise; that is, there exists $w^{\prime} \in W$ such that $w^{\prime} \notin \mu_{W}(\mu(W))$. Since $\mu_{W}(W)=\mu(W), \mu_{W}\left(w^{\prime}\right)=\emptyset$. Thus,

$$
\sum_{f \in \mu(W)}\left|\mu_{W}(f)\right|<|W|=\sum_{f \in \mu(W)}|\mu(f)|,
$$

where the equality follows from (1). Thus, there exists $\bar{f} \in \mu(W)$ such that $\left|\mu_{W}(\bar{f})\right|<$ $|\mu(\bar{f})| \leq q_{\bar{f}}$, implying that we can find $\bar{w} \in \mu(\bar{f}) \backslash \mu_{W}(\bar{f})$. By $(1), \bar{f}=\mu(\bar{w}) P(\bar{w}) \mu_{W}(\bar{w})$ and by the quota $q_{\bar{f}}$-separability of $P(\bar{f})$ and the individual rationality of $\mu,(\bar{f}, \bar{w})$ blocks $\mu_{W}$, in contradiction with its stability. Thus,

$$
\mu_{W}(\mu(W))=W
$$

Claim B is proved.

Consider now the last step of the deferred-acceptance algorithm where workers make offers (and which yields, as its outcome, matching $\mu_{W}$ ). Let $\bar{w}$ be a worker who makes an offer to an acceptable firm $f$ in the last step of the algorithm. If $f$ rejects some worker $w^{\prime}$, then this worker is unmatched in $\mu_{W}$, since we are considering the last step of the algorithm. This contradicts Claim B. Thus, $f$ does not reject any worker. By the substitutability of $P(f)$ and $(1)$,

$$
\mu_{W}(f)=C h\left(S_{f, \bar{w}} \cup\{\bar{w}\}, P(f)\right)=C h\left(S_{f, \bar{w}}, P(f)\right) \cup\{\bar{w}\} \supseteq \mu(f) \cup\{\bar{w}\},
$$

where $S_{f, \bar{w}}=\{w \in W \backslash\{\bar{w}\} \mid w$ makes an offer to $f$ during the algorithm $\}$. The last inclusion holds because $\mu(f) \subseteq S_{f, \bar{w}}((1)$ implies that all $w \in \mu(f)$ make an offer to $f$ during the algorithm). Thus,

$$
\mu_{W}(f) \supseteq \mu(f) \cup\{\bar{w}\} .
$$

Then, since $\bar{w} \notin \mu(f)$, we have that $q_{f} \geq\left|\mu_{W}(f)\right| \geq|\mu(f)|+1$ and thus, $|\mu(f)|<q_{f}$. If $|\mu(f)|>0$, then we can find $w \in \mu(f) \cap \mu_{W}(f)$, contradicting (1). Thus, $|\mu(f)|=0$ which implies that $\mu(f)=\emptyset$. The inclusion in (7) says that $\bar{w} \in \mu_{W}(f)$ holds. Hence, we obtain a contradiction, since $f \in \mu_{W}(W)$ and $f \notin \mu(W)$ imply that Claim A does not hold.

Proof of the Theorem The statement of the Theorem follows immediately from the following Claim.

Claim Let $P$ be a substitutable and quota $q$-separable preference profile and let $\widehat{P}$ differ from $P$ in that some nonempty subset $\widehat{W}$ of workers have different preference relations. Then, there is no matching $\mu \in S(\widehat{P})$ such that $\mu P(w) \mu_{W}$ by all $w \in \widehat{W}$. 
Proof Assume otherwise; that is, there exists a nonempty subset of workers $\widehat{W}$ and a matching $\mu \in S(\widehat{P})$ such that for all $w \in \widehat{W}, \mu(w) P(w) \mu_{W}(w)$. We first show that $\mu \in$ $I R(P)$. Since $\mu \in S(\widehat{P})$ and $\widehat{P}(i)=P(i)$ for all $i \in(F \cup W) \backslash \widehat{W}$, then $\mu$ is individually rational for all $i \notin \widehat{W}$. Moreover, if $w \in \widehat{W}$ then $\mu(w) P(w) \mu_{W}(w) R(w) \emptyset$. Hence, $\mu \in$ $I R(P)$. Since all matchings $\mu^{\prime} \in S(P)$ have the property that $\mu_{W} R(w) \mu^{\prime}$ for all $w \in W$ and there exists at least one $w \in \widehat{W}$ with $\mu(w) P(w) \mu_{W}(w)$, we conclude that $\mu \notin S(P)$. Since $\widehat{W} \neq \emptyset$ and because $\emptyset \neq \widehat{W} \subseteq W^{\prime}=\left\{w \in W \mid \mu(w) P(w) \mu_{W}(w)\right\}$ we can apply the Blocking Lemma. Thus, there is a pair $(\bar{f}, \bar{w})$, where $\bar{f} \in \mu\left(W^{\prime}\right)$ and $\bar{w} \in W \backslash W^{\prime}$, that blocks $\mu$ in $P$, but $\bar{w} \in W \backslash W^{\prime}$ implies that $\widehat{P}(\bar{w})=P(\bar{w})$. Thus, $(\bar{f}, \bar{w})$ blocks $\mu$ in $\widehat{P}$, contradicting that $\mu \in S(\widehat{P})$.

\section{References}

[1] S. Barberà, H. Sonnenschein, and L. Zhou. "Voting by Committees", Econometrica 59, 595-609 (1991).

[2] G. Demange, D. Gale, and M. Sotomayor. "A Further Note on the Stable Matching Problem", Discrete Applied Mathematics 16, 217-222 (1987).

[3] L. Dubins and D. Freedman. "Machiavelli and the Gale-Shapley Algorithm", American Mathematical Monthly 88, 485-494 (1981).

[4] B. Dutta and J. Massó. "Stability of Matchings When Individuals Have Preferences over Colleagues", Journal of Economic Theory 75, 464-475 (1997).

[5] D. Gale and L. Shapley. "College Admissions and the Stability of Marriage", American Mathematical Monthly 69, 9-15 (1962).

[6] A. Kelso and V. Crawford. "Job Matching, Coalition Formation, and Gross Substitutes", Econometrica 50, 1483-1504 (1982).

[7] R. Martínez, J. Massó, A. Neme, and J. Oviedo. "Single Agents and the Set of Manyto-one Stable Matchings", Journal of Economic Theory 91, 91-105 (2000).

[8] R. Martínez, J. Massó, A. Neme, and J. Oviedo. "On the Lattice Structure of the Set of Stable Matchings for a Many-to-one Model", Optimization 50, 439-457 (2001). 
[9] R. Martínez, J. Massó, A. Neme, and J. Oviedo. "The Blocking Lemma for a Manyto-one Matching Model," http://pareto.uab.es/jmasso/BlockingLemma.pdf (2004).

[10] S. Mongell and A. Roth. "Sorority Rush as a Two-Sided Matching Mechanism", American Economic Review 81, 441-464 (1991).

[11] A. Romero-Medina. "Implementation of Stable Solutions in a Restricted Matching Market", Review of Economic Design 3, 137-147 (1998).

[12] A. Roth. "The Economics of Matching: Stability and Incentives", Mathematics of Operations Research 7, 617-628 (1982).

[13] A. Roth. "The Evolution of the Labor Market for Medical Interns and Residents: A Case Study in Game Theory", Journal of Political Economy 92, 991-1016 (1984).

[14] A. Roth. "Conflict and Coincidence of Interest in Job Matching: New Results and Open Questions", Mathematics of Operations Research 10, 379-389 (1985).

[15] A. Roth. "On the Allocation of Residents to Rural Hospitals: A General Property of Two-Sided Matching Markets", Econometrica 54, 425-427 (1986).

[16] A. Roth. "New Physicians: A Natural Experiment in Market Organization", Science 250, 1524-1528 (1990).

[17] A. Roth. "A Natural Experiment in the Organization of Entry-Labor Markets: Regional Markets for New Physicians and Surgeons in the United Kingdom", American Economic Review 81, 415-440 (1991).

[18] A. Roth and M. Sotomayor. Two-sided Matching: A Study in Game-Theoretic Modeling and Analysis. Cambridge University Press, Cambridge, England. [Econometrica Society Monographs No. 18] (1990).

[19] A. Roth and X. Xing. "Jumping the Gun: Imperfections and Institutions Related to the Timing of Market Transactions", American Economic Review 84, 992-1044 (1994).

[20] T. Sönmez. "Strategy-proofness in Many-to-one Matching Problems", Economic Design 1, 365-380 (1996). 